\title{
Achieving anisotropy in metamaterials made of dielectric cylindrical rods
}

\author{
Peng, Liang; Ran, Lixin; Mortensen, Asger
}

Published in:

Applied Physics Letters

Link to article, DOI:

$10.1063 / 1.3453446$

Publication date:

2010

Document Version

Publisher's PDF, also known as Version of record

Link back to DTU Orbit

Citation (APA):

Peng, L., Ran, L., \& Mortensen, A. (2010). Achieving anisotropy in metamaterials made of dielectric cylindrical rods. Applied Physics Letters, 96(24), 241108. https://doi.org/10.1063/1.3453446

\section{General rights}

Copyright and moral rights for the publications made accessible in the public portal are retained by the authors and/or other copyright owners and it is a condition of accessing publications that users recognise and abide by the legal requirements associated with these rights.

- Users may download and print one copy of any publication from the public portal for the purpose of private study or research.

- You may not further distribute the material or use it for any profit-making activity or commercial gain

- You may freely distribute the URL identifying the publication in the public portal

If you believe that this document breaches copyright please contact us providing details, and we will remove access to the work immediately and investigate your claim 


\title{
Achieving anisotropy in metamaterials made of dielectric cylindrical rods
}

\author{
L. Peng, ${ }^{1,2}$ Lixin Ran, ${ }^{1, a)}$ and N. A. Mortensen ${ }^{2, b)}$ \\ ${ }^{1}$ Department of Information and Electronic Engineering, Zhejiang University, Hangzhou 310027, \\ People's Republic of China \\ ${ }^{2}$ Department of Photonics Engineering, Technical University of Denmark, DTU-building 345 west, \\ DK-2800 Kongens Lyngby, Denmark
}

(Received 21 April 2010; accepted 23 May 2010; published online 16 June 2010)

\begin{abstract}
We show that anisotropic negative effective dispersion relation can be achieved in pure dielectric rod-type metamaterials by turning from the symmetry of a square lattice to that of a rectangular one. Theoretical predictions and conclusions are verified by both numerical calculations and computer based simulations. The proposed anisotropic metamaterial, is used to construct a refocusing slab lens and a subdiffraction hyperlens. The all-dielectric origin makes it more straightforward to address loss and scaling, thus facilitating future applications in both the terahertz and optical range. (C) 2010 American Institute of Physics. [doi:10.1063/1.3453446]
\end{abstract}

In recent years, metamaterials (MMs) made of a stack of small structural elements have attracted substantial attention. The inherent ability of high-index structures to support strong subwavelength resonances allows the associated MMs to behave as effective media with various unusual macroscopic electromagnetic (EM) properties, such as negative permittivity/permeability and negative refraction. ${ }^{1-3}$ With peculiar collective EM characteristics, MMs can be used to fabricate innovative devices like subwavelength refocusing lenses ${ }^{4}$ and invisibility cloaks. ${ }^{5}$

Currently, one of the most important issues on MMs is to scale the MM unit cells down in size to allow operating a yet higher frequencies, i.e., terahertz and optical regions. ${ }^{6,7}$ In this context, dielectrics rather than metals might be preferred by MMs, due to the loss issue of metals at high frequencies. Till now, great efforts have been devoted to developing homogenization theory for the pure dielectric MMs. Rod-type dielectric resonators with high permittivity have been used to fabricate left-handed (LH) MMs in the microwave range and the terahertz range. ${ }^{89}$ Recent scaling analysis have also pointed out that LH behaviors at optical frequencies would also be possible by use of rod-type MMs made of silicon. ${ }^{10}$ Rod-type dielectric MMs with an isotropic density of rods (ordered or disordered) will commonly possess an isotropic dispersion in the two dimensions. ${ }^{8,10}$ While isotropic dispersion is advantageous in a number of cases, there is however also a strong call for realizing anisotropic MMs for practical applications, as is in general required for a transformation medium.

In this paper, we report on both theoretical and numerical studies of the dielectric rod-type MMs with asymmetrical shaped unit cells. General closed-form expressions for the effective constitutive parameters are obtained from a macroscopic viewpoint. We show that the dispersion of such dielectric MMs would be naturally spatially anisotropic with negative refraction, supported by parameters extracted from numerical computation and EM phenomena in other simulations. By using this kind of anisotropic rod-type MMs, a farfield slab lens with different refocusing distance is conveniently realized. Moreover, this kind of MMs can also be

\footnotetext{
${ }^{a)}$ Electronic mail: ranlx @zju.edu.cn.

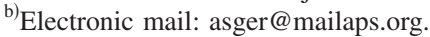

implemented to fabricate a slab-type hyperlens with subdiffraction limit. Both of these designs are supported by fullwave simulations.

We start by considering the array composed of standard cylindrical resonators, as shown in Fig. 1(a). For simplicity, we emphasize the ordered case but the composite could be disordered, since the low-frequency macroscopic EM properties of this kind of MM do not rely on band structure phenomena, but the low-lying Mie resonances of the isolated cylinders. ${ }^{8,10}$ As discussed in Ref. 8 , the displacement current distribution inside the cylinder in the basic two Mie resonance modes would be similar to that of the conductive current in metallic rod and split-ring resonator, thus also supporting effective negative permittivity/permeability of the MM. When the dielectric cylinders are arranged, in a regular lattice or in a disordered fashion, the whole composite will exhibit isotropic EM responses. Spatial anisotropy may be introduced by relaxing this isotropic density, thus breaking the effective rotational symmetry of the long-wavelength effective medium.

Here, without loss of generality, rod-type dielectric MM would have a rectangular shaped unit cell, as shown in Fig. 1(b). Basically, as an effective medium, the MM's unit cell dimensions have to be much smaller than the wavelength in free space. ${ }^{8}$ The effective parameters, i.e., $\boldsymbol{\varepsilon}_{\text {eff }}$ and $\boldsymbol{\mu}_{\text {eff }}$, are defined in Ref. 8 as $\langle\boldsymbol{D}\rangle=\boldsymbol{\varepsilon}_{\text {eff }}\langle\boldsymbol{E}\rangle$ and $\langle\boldsymbol{B}\rangle=\boldsymbol{\mu}_{\text {eff }}\langle\boldsymbol{H}\rangle$. From Mie scattering theory, all the correlated fields can formally be expressed by superposition of vector cylindrical waves. ${ }^{11}$ Here, when the exciting electric field is polarized along the
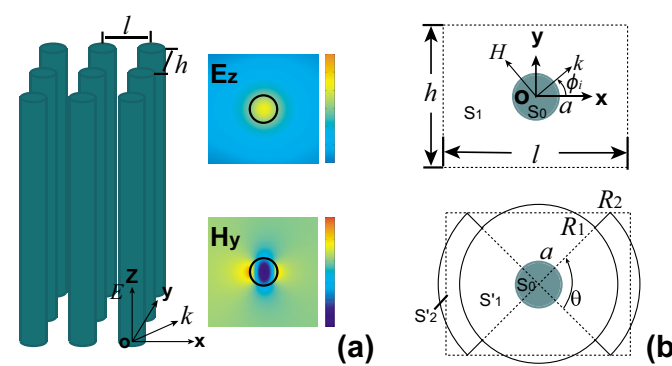

FIG. 1. (Color online) (a) The configuration of rod-type dielectric MMs The two insets show the $\mathrm{E} / \mathrm{H}$ field distribution in the first two resonance modes. (b) The cross section schematic of rod-type MM unit cell (upper) and its simplified integral region (lower). 
$z$-axis, see Fig. 1(a), the practical geometry of the lattice complicates a closed-form analytical solution for the averaging over the rectangular shaped area $S_{1}$. For the ease of analysis, we approximate the integral over the rectangular domain by performing the integrals on an equivalent region formed by $S_{0}, S_{1}^{\prime}$, and $S_{2}^{\prime}$, see the lower part of Fig. 1(b). In this way simplify the problem, yet still capturing the correct physics. With these approximations and regardless of the high-order scattering, straightforward algebraic operation would eventually give

$$
\frac{\varepsilon_{\mathrm{eff}, z}}{\varepsilon_{0}}=\frac{S-\pi a^{2}}{S}\left[1-\frac{2 i \varepsilon_{p} J_{1}\left(k_{p} a\right)}{k_{p} c_{0}} \frac{E_{0}}{X_{z}}\right],
$$

with

$$
\begin{aligned}
X_{z} \approx & \pi a_{0}^{(N)}\left[\rho H_{1}^{(1)}(k \rho)\right]_{a}^{R_{1}}+\pi E_{0}\left[\rho J_{1}(k \rho)\right]_{a}^{R_{1}} \\
& +\theta a_{0}^{(N)}\left[\rho H_{1}^{(1)}(k \rho)\right]_{R_{1}}^{R_{2}}+\theta E_{0}\left[\rho J_{1}(k \rho)\right]_{R_{1}}^{R_{2}}, \\
c_{n}= & \sqrt{\left(\varepsilon_{p} / \mu_{p}\right)} H_{n}^{(1)}(k a) J_{n}^{\prime}\left(k_{p} a\right)-H_{n}^{\prime(1)}(k a) J_{n}\left(k_{p} a\right)
\end{aligned}
$$

and $a_{n}^{(N)}=\left(E_{0} / c_{n}\right)\left[J_{n}^{\prime}(k a) J_{n}\left(k_{p} a\right)-\sqrt{\left(\varepsilon_{p} / \mu_{p}\right)} J_{n}(k a) J_{n}^{\prime}\left(k_{p} a\right)\right]$. $a$, $R_{1}, R_{2}$, and $\theta$ are parameters depicted in Fig. 1(b). Similarly, we find

$$
\begin{aligned}
\frac{\mu_{\mathrm{eff}, x(y)}}{\mu_{0}}= & \frac{S-\pi a^{2}}{S}\left\{1+\pi a\left[J_{1}(k a)\right.\right. \\
& \left.\left.+H_{1}^{(1)}(k a) \frac{a_{1}^{(N)}}{E_{0}}\right] \frac{E_{0}}{Y_{x(y)}}\right\},
\end{aligned}
$$

with

$$
\begin{aligned}
Y_{x(y)}= & \pi a_{1}^{(N)}\left[\rho H_{1}^{(1)}(k \rho)\right]_{a}^{R_{1}}+\pi E_{0}\left[\rho J_{1}(k \rho)\right]_{a}^{R_{1}} \\
& +E_{0} / k(\theta \pm \sin \theta)\left[J_{0}(k \rho)\right]_{R_{1}}^{R_{2}} \\
& -E_{0} / k(\theta \mp \sin \theta)\left[J_{0}(k \rho)\right]_{R_{1}}^{R_{2}} \\
& +E_{0}(\theta \mp \sin \theta)\left[\rho J_{1}(k \rho)\right]_{R_{1}}^{R_{2}} \\
& +a_{1}^{(N)} / k(\theta \pm \sin \theta)\left[H_{0}^{(1)}(k \rho)\right]_{R_{1}}^{R_{2}} \\
& -a_{1}^{(N)} / k(\theta \mp \sin \theta)\left[H_{0}^{(1)}(k \rho)\right]_{R_{1}}^{R_{2}} \\
& +a_{1}^{(N)}(\theta \mp \sin \theta)\left[\rho H_{1}^{(1)}(k \rho)\right]_{R_{1}}^{R_{2}} .
\end{aligned}
$$

As an example, we assume that the dielectric rods are made of $\mathrm{Ba}_{0.5} \mathrm{Sr}_{0.5} \mathrm{TiO}_{3}$ (BST) ceramic, whose dielectric constant is around 600 in the microwave range. ${ }^{8}$ It was shown that a MM made from BST rods has a double negative band. ${ }^{8,10}$ Here the dimensions of the asymmetric lattice are $l=7.4 \mathrm{~mm}$ and $h=7 \mathrm{~mm}$, which will support an anisotropic dispersion relation. Figure 2(a) shows the calculated effective refractive index (RI) $n_{\text {eff }}=\sqrt{\varepsilon_{\text {eff }}} \sqrt{\mu_{\text {eff }}}$ in both $x$ and $y$ directions. Now we focus our eyes on those frequencies at which the absolute value of the refractive index is small, see the inset of Fig. 2(a). The difference between $n_{x}$ and $n_{y}$ indicates the anisotropic property of this MMs. In addition, we have also performed wave simulations and extract the effective RI by a retrieval method. ${ }^{12}$ The extracted data is shown in Fig. 2(b). Obviously, Fig. 2(a) is qualitatively consistent with Fig. 2(b).

Formally, the dispersion relation of an effective medium could be written in an analytical form $\left(k_{x}^{2} / n_{x}^{2}\right)+\left(k_{y}^{2} / n_{y}^{2}\right)=k_{0}^{2}$,

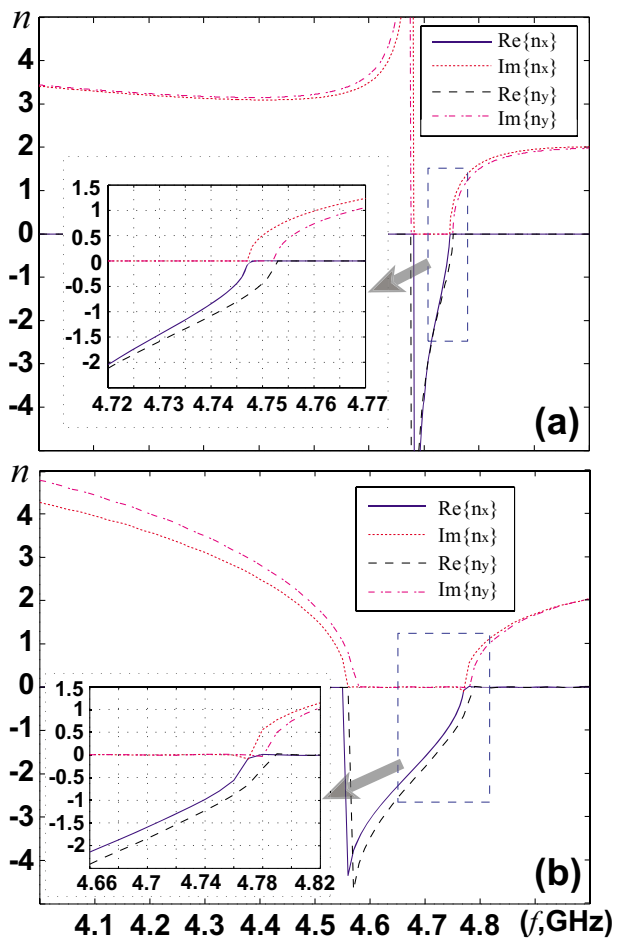

FIG. 2. (Color online) Effective refractive index in different directions of anisotropic rod-type MM. (a) Theoretical estimation. (b) Data extracted from numerical simulation. In the calculation and simulation, $a=1 \mathrm{~mm}$, $l=7.4 \mathrm{~mm}, h=7 \mathrm{~mm}$, and the relative dielectric constant of the rod is 600 .

with $n_{x}$ and $n_{y}$ being the RI in $x$ and $y$ directions, respectively. Though the different effective RI associated with different directions is responsible for the anisotropic EM response, it is quite difficult to verify the complete dispersion curve by a retrieval method ${ }^{12}$ because of the rotational asymmetry. Here, we instead illustrate the anisotropic property, by the example of a device which requires an anisotropic MM, i.e., refocusing of a point source by a slab lens.

As an effect of a compensating bilayer, a general focusing slab lens can be realized by anisotropic negative RI MMs whose dispersion relation has a form of $k_{x}^{2}+\left(k_{y}^{2} / r^{2}\right)=k_{0}^{2}$, where $r=\left(d^{\prime}-d\right) / d$ is relating to the distance between source and its image $\left(d^{\prime}\right)$ versus width of the slab lens $(d) .{ }^{13}$ By using our MM, such a slab lens would be easily constructed. The parameters are $\varepsilon=600$ and $a=1 \mathrm{~mm}$ for the BST rods, and $l=h=7 \mathrm{~mm}$ for the first lens. Because of the isotropic dispersion relation, a refocused image can be found at a distance $d^{\prime}=2 d$, see the power flow density distribution (PFDD) in Fig. 3(a). Next, we keep $l$ unchanged but vary $h$ in the $y$ direction. In case (I), $h$ is increased to $7.4 \mathrm{~mm}$, and from the PFDD in Fig. 3(c) we see that the source is refocused again at $d^{\prime}<2 d$ with a little down shifting of operation frequency. In case (II), $h$ is decreased to $6.4 \mathrm{~mm}$, in which case the refocused image is found at a distance $d^{\prime}>2 d$. For comparison, we have also simulated the PFDD of refocusing by an ideal negative index anisotropic slab lens, see Fig. 3(b) (isotropic material with $n \approx-1$ ), and panels in Figs. 3(d) and 3(f) are for an anisotropic material with $k_{x}^{2}+1.4^{2} k_{y}^{2}=k_{0}^{2}$ and $k_{x}^{2}+\left(k_{y}^{2} / 1.5^{2}\right)=k_{0}^{2}$.

Clearly, from Fig. 3, the refocusing of a MM slab lens is governed by the effective constitutive relations. However, it is very hard to obtain subwavelength resolution in such an imaging case, since it generally mismatches the free space. ${ }^{8}$ Fortunately, there is an alternative way to achieve subwave- 


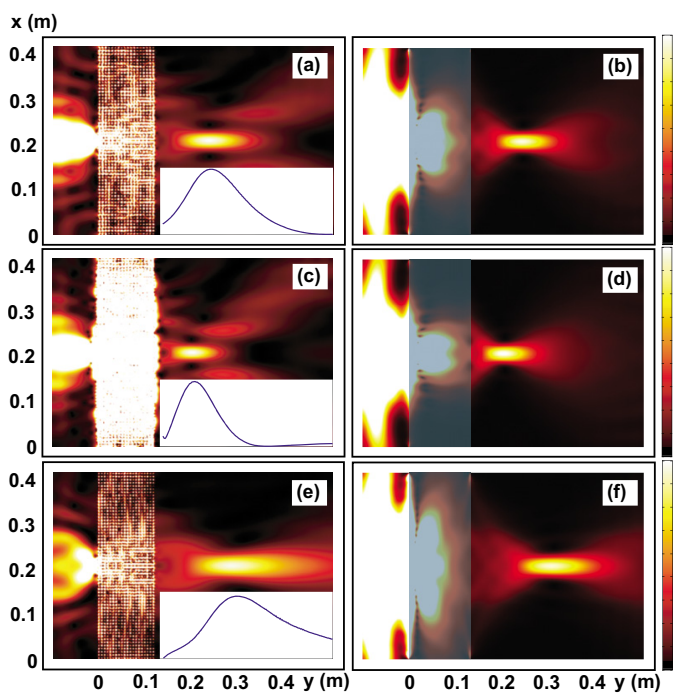

FIG. 3. (Color online) PFDD in the refocusing by three different slab lenses made of pure dielectric MMs. The periodicities in $x$ and $y$ directions are (a) $7 \times 7 \mathrm{~mm}^{2}$, (c) $7 \times 7.4 \mathrm{~mm}^{2}$, and (e) $7 \times 6.4 \mathrm{~mm}^{2}$; the widths of the three superlens are around $0.128 \mathrm{~m}$. In the three simulations, the operation frequencies change slightly, respectively. The insets of (a), (c), and (e) are the cross section of the PFDD at $y=0.21 \mathrm{~m}$. Subfigures (b), (d), and (f) are the PFDD in the refocusing by three ideal anisotropic slab lens with $0.128 \mathrm{~m}$ width.

length resolution with this MMs. From Fig. 2, the effective refractive index in one direction will turn imaginary beyond the upper bound of the double negative band, i.e., $n_{x}$ is imaginary at $4.78 \mathrm{GHz}$ in Fig. 2(b), while $n_{y}$ stays real. Thus, the MMs' effective dispersion would be hyperbolic in a narrow frequency band, e.g., from 4.77 to $4.79 \mathrm{GHz}$ in Fig. 2(b). Thus, this MM can be used to form a hyperlens which can distinguish sources with subwavelength separation placed close to the interface. ${ }^{14-17}$

Again, BST cylinders are adopted to construct a MM slab, see Fig. 4(a). In the simulation, two point sources with a separation of $16 \mathrm{~mm}$ are placed at $y=-0.005 \mathrm{~m}$. The simulated PFDD around $4.81 \mathrm{GHz}$ is shown in Fig. 4(a), and indeed we observe two images. For a clearer visualization, panel in Fig. 4(b) shows cross-section cuts of the PFDD at $y=-0.005 \mathrm{~m}$ and $y=0.128 \mathrm{~m}$. Keeping the radiation wavelength in mind, the ability to distinguish the two images indicates subdiffraction imaging.

In conclusion, we have proposed a way to design anisotropic negative-index MMs by means of pure dielectric rodtype rectangular lattices. Results are supported by a homogenization approach as well as by numerical calculation and simulations. From our theoretical analysis, the effective dispersion is represented by closed form expressions like a realistic anisotropic media existing in nature. With the anisotropic MMs proposed here, we have constructed a far field refocusing slab lens and a subdiffraction hyperlens. Since the effective constitutive parameters play the key role in achieving the EM phenomena, anisotropic rod-type MMs could also be used to some other applications, e.g., constructing an invisibility cloak or transformation medium. Moreover, our study in this paper points out flexible design of dielectric rod-type MMs, which avoids the loss and scaling issue of
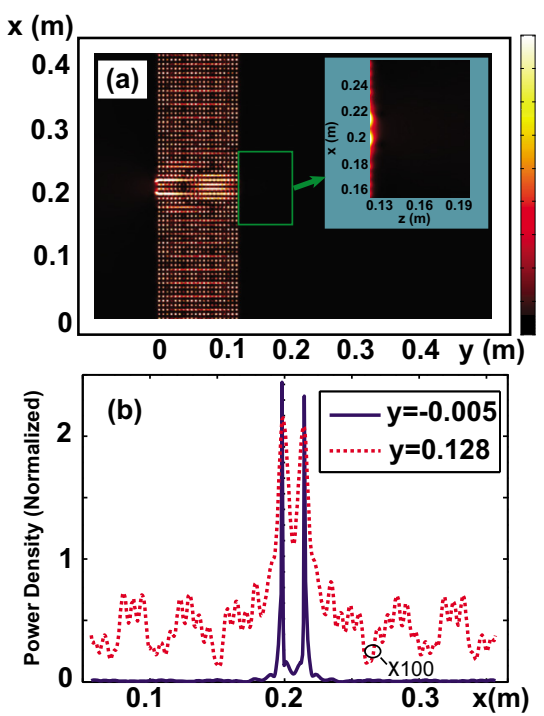

FIG. 4. (Color online) Subdiffraction by a slab-shape hyperlens made of rod-type dielectric MM. (a) PFDD; (b) cross section draws of PFDD at $y$ $=-0.005 \mathrm{~m}$ and $y=0.128 \mathrm{~m}$. In the simulation, $f \approx 4.81 \mathrm{GHz}, a=1 \mathrm{~mm}$, $l=7 \mathrm{~mm}, h=6.4 \mathrm{~mm}$, while the interval of the two sources is $16 \mathrm{~mm}$. In (b), the data at $y=0.128 \mathrm{~m}$ is normalized by a factor of 100 to have a better comparison.

metallic structures in realizing negative properties, indicating many potential applications in terahertz and optical range in future.

This work was sponsored by NSFC (Grant No. 60531020), 863 Project (Grant No. 2009AA01Z227), Program for New Century Excellent Talents (Grant No. NCET07-0750), the National Key Laboratory Foundation (Nos. 9140C5304020901 and 9140C5304020704), and the Danish HTF (Grant No. 004-2007-1).

${ }^{1}$ J. B. Pendry, A. J. Holden, W. J. Stewart, and I. Youngs, Phys. Rev. Lett. 76, 4773 (1996).

${ }^{2}$ J. B. Pendry, A. J. Holden, D. J. Robbins, and W. J. Stewart, IEEE Trans. Microwave Theory Tech. 47, 2075 (1999).

${ }^{3}$ R. A. Shelby, D. Smith, and S. Schultz, Science 292, 77 (2001).

${ }^{4}$ J. B. Pendry, Phys. Rev. Lett. 85, 3966 (2000).

${ }^{5}$ J. B. Pendry, D. Schurig, and D. R. Smith, Science 312, 1780 (2006).

${ }^{6}$ N. Liu, H. C. Guo, L. W. Fu, S. Kaiser, H. Schweizer, and H. Giessen, Nat. Mater. 7, 31 (2008).

${ }^{7}$ J. Valentine, S. Zhang, T. Zentgraf, E. Ulin-Avila, D. A. Genov, G. Bartal, and X. Zhang, Nature (London) 455, 376 (2008).

${ }^{8}$ L. Peng, L. Ran, H. Chen, H. Zhang, J. A. Kong, and T. M. Grzegorczyk, Phys. Rev. Lett. 98, 157403 (2007).

${ }^{9}$ J. A. Schuller, R. Zia, T. Taubner, and M. L. Brongersma, Phys. Rev. Lett. 99, 107401 (2007).

${ }^{10}$ K. Vynck, D. Felbacq, E. Centeno, A. I. Căbuz, D. Cassagne, and B. Guizal, Phys. Rev. Lett. 102, 133901 (2009).

${ }^{11}$ L. Tsang, J. A. Kong, and K. H. Ding, Scattering of Electromagnetic Wave (Wiley, New York, 2000), Vol. 1.

${ }^{12}$ X. Chen, T. M. Grzegorczyk, B. I. Wu, J. J. Pacheco, and J. A. Kong, Phys. Rev. E 70, 016608 (2004).

${ }^{13}$ D. Schurig and D. R. Smith, New J. Phys. 7, 162 (2005).

${ }^{14}$ A. Salandrino and N. Engheta, Phys. Rev. B 74, 075103 (2006).

${ }^{15}$ Z. Jacob, L. V. Alekseyev, and E. Narimanov, Opt. Express 14, 8247 (2006).

${ }^{16}$ I. I. Smolyaninov, Y.-J. Hung, and C. C. Davis, Science 315, 1699 (2007).

${ }^{17}$ Z. Liu, H. Lee, Y. Xiong, C. Sun, and X. Zhang, Science 315, 1686 (2007) 